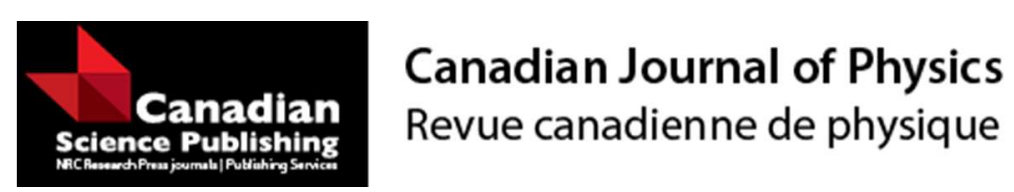

\title{
Dynamical analysis of autonomous system in entropic cosmology
}

\begin{tabular}{|r|l|}
\hline Journal: & Canadian Journal of Physics \\
\hline Manuscript ID & cjp-2015-0785.R1 \\
\hline Manuscript Type: & Article \\
\hline Date Submitted by the Author: & 31 -Jan-2016 \\
\hline Complete List of Authors: & Li, Yu; Dalian Maritime University, Department of Physics \\
\hline Keyword: & $\begin{array}{l}\text { entropic cosmology, autonomous system, stability properties, fixed points, } \\
\text { entropic-force terms }\end{array}$ \\
\hline & \\
\hline
\end{tabular}

SCHOLARONE $^{\text {In }}$

Manuscripts 


\title{
Dynamical analysis of autonomous system in entropic cosmology
}

\author{
$\mathrm{Yu} \mathrm{Li*}$ \\ Department of Physics, Dalian Maritime University, Dalian 116026, China
}

(Dated: February 18, 2016)

\begin{abstract}
The entropic cosmology model is an alternative method to explain the accelerated expansion of the universe. In this paper, we discuss the dynamical system in two types of entropic cosmology model, i.e. $\Lambda(t)$ type and BV type. We found that, the stability properties of fixed points are affected by $H^{2}$ term, while the $H$ term and constant term have no influence on stability properties of fixed points. We also found that, the dynamical properties of $\mathrm{C}$-version model are same as $\mathrm{H}$-version model.

Keyword: entropic cosmology; dark energy; dynamical system.

PACS numbers: 98.80.-k, 95.36.+x
\end{abstract}

\section{INTRODUCTION}

Observations indicate that the expansion rate of universe is accelerating [1-5]. There are several methods to explain the accelerated expansion of the late universe[6]. The most important method is the introduction of dark energy $[7,8]$. The simplest dark energy is cosmology constant $\Lambda$. However, the energy density of cosmology constant which is needed by cosmologists is much smaller than the energy of the vacuum in quantum theory. The commonly used method is to introduce scalar fields to replace the cosmological constant.

Recently, an alternative method, named entropic cosmology model, is proposed to explain the accelerated expansion of the universe $[9,10]$. The basic idea of entropic cosmology is to consider the entropic-force terms which are derived from the usually neglected surface terms on the horizon of the universe [9]. (The idea of the entropicforce term on the horizon can explain the accelerating universe in [9] should be distinguished from the idea that gravity itself is an entropic force [11].Although they use the same terms, they are two very different ideas.) Different entropic-force terms are derived from different classes of entropy. In original entropic cosmology model $[12,13]$, the entropic terms include $H^{2}$ terms and $\dot{H}$ terms. $H^{2}$ terms are derived from Bekenstein entropy (area entropy), where $H$ is Hubble parameter. Further research shows that, the background equations with $H^{2}$ term and $\dot{H}$ term can be made equivalent to a model with a dark energy component[14]. Other works [13, 15, 16] suggest that, the behavior of $H^{2}$ terms and $\dot{H}$ terms are found to be same, therefore the $\dot{H}$ term can be neglected [15]. Besides the $H^{2}$ terms, there are $H$ terms in entropic-force terms and they are derived from volume entropy [15]. Moreover, in [16], the author shows that the $H^{2}$ terms do not describe cosmological fluctuations properly without the inclusion of constant terms which are derived from entropy proportional to the square of area entropy[15], therefore it is necessary to introduce constant entropic-force terms.

* leeyudlmu@gmail.com
Moreover, in some discussions [17], $H^{2}$ terms have been neglected, while $H$ terms and constant terms are considered separately. In this case, the entropic cosmology also can be divided into two categories, i.e. H-version and C-version.

According to different forms of evolution equations, there are two types of entropic cosmology model [17]. The first type is so-called $\Lambda(t)$ type, which is similar to $\Lambda(t) \mathrm{CDM}[18-25]$. The entropic-force terms are included both in Friedmann equation and acceleration equation in this type. The second type is BV type, which is similar to bulk viscous models[26-41]. In this type, the entropicforce terms appear only in acceleration equation.

The entropic cosmology model, which is different from dark energy, is another method to explain the accelerated expansion of the universe. Therefore, it is necessary to study the dynamical properties of entropic cosmology model.

However, this does not mean that dark energy is not necessary in entropic cosmology model. In addition to explaining the accelerated expansion of the universe, dark energy is an indispensable component of the universe. Measurements of anisotropy of cosmology microwave background indicate that, the space of universe is flat, which means that $\Omega_{0} \approx 1$. Meanwhile, a large number of observational facts show that, the radiation and nonrelativistic matter (mainly baryons and nonbaryonic dark matter) only contribute about $30 \%$ of $\Omega_{0}$, which means that dark energy should contribute about $70 \%$ of $\Omega_{0}$. Therefore, in this paper, we consider the entropic comology model with dark energy. We introduce a scalar field in two types of entropic cosmology model and discuss the influence of different entropic-force terms on the dynamical properties of autonomous system in entropic cosmology.

The paper is organized as follows: In Sec.II we sum up on the dynamical equations of entropic cosmology, in which we introduce a scalar field $\phi$. We discuss the autonomous systems in $\Lambda(t)$ type model in Sec.III and BV type in Sec.IV. The summary and discussion is in Sec.V. 


\section{DYNAMICAL SYSTEM IN ENTROPIC COSMOLOGY}

The Friedmann equation of a homogeneous, isotropic, spatially flat entropic cosmology is [17]

$$
\frac{\dot{a}}{a}=H^{2}=\frac{\kappa^{2}}{3} \rho(t)+f(t)
$$

and the acceleration equation is [17]

$$
\frac{\ddot{a}}{a}=\dot{H}+H^{2}=-\frac{\kappa^{2}}{6}[\rho(t)+p(t)]+g(t)
$$

where $\kappa^{2}=8 \pi G$, and $G$ is gravitational constant. $H$ is Hubble parameter and defined by

$$
H(t) \equiv \frac{d a(t) / d t}{a(t)}=\frac{\dot{a}(t)}{a(t)}
$$

$f(t)$ and $g(t)$ are entropic-force terms. In general model of entropic cosmology, the form of entropic-force terms are $[17]$

$$
\begin{gathered}
f(t)=\alpha_{1} H^{2}+\alpha_{3} H_{0} H+\alpha_{4} H_{0}^{2} \\
g(t)=\beta_{1} H^{2}+\beta_{3} H_{0} H+\beta_{4} H_{0}^{2}
\end{gathered}
$$

where $H_{0}$ is the Hubble parameter at the present time.

The coefficients $\alpha_{1}, \alpha_{3}, \alpha_{4}$ and $\beta_{1}, \beta_{3}, \beta_{4}$ are dimensionless constants. In $[10,12]$, it was argued that the value of coefficients is likely

$$
\alpha_{1}=\beta_{1}=\frac{3}{2 \pi}
$$

In this paper, we do not restrict the value of this coefficients, i.e. we discuss the general case of entropic cosmology.

One can obtain the continuity equation from Eq. $(1,2)$ $[17,42]$

$$
\dot{\rho}+3 \frac{\dot{a}}{a}(\rho+p)=Q
$$

where

$$
Q=\frac{6}{\kappa^{2}} H\left[-f(t)-\frac{\dot{f}(t)}{2 H}+g(t)\right]
$$

$Q$ can be seen as the energy exchange between different component of universe $[17,43]$. We consider the cosmology model which is filled by some positive pressure matter with density $\rho_{m}$ and a scalar field $\phi$ with exponential form potential $V=V_{0} \exp (-\lambda \kappa \phi)$.

It should be noted that the exponential form potential itself can be used to explain the accelerating expansion of the universe. However, we are still using the exponential form potential in entropic cosmology model based on the following two reasons: First, such potentials arise naturally in supergravity models [44], and it play an important role in driving a period of early inflation [45], ekpyrotic or pre big bang collapse [46]. Second: The purpose of this paper is to study the influence of various entropy-force terms on the dynamical properties of the universe. Meanwhile, the exponential form potential is one of the most widely used in the cosmological model. Therefore the using of such potentials in entropic cosmology model makes it convenient for us to compare the conclusions in this paper with the conclusions of other cosmology models.

To simplify the discussion, we restrict the value of $\lambda$ to positive. So we have

$$
\begin{aligned}
& \rho=\rho_{m}+\rho_{\phi}=\rho_{m}+\frac{\dot{\phi}^{2}}{2}+V \\
& p=p_{m}+p_{\phi}=p_{m}+\frac{\dot{\phi}^{2}}{2}-V
\end{aligned}
$$

and the continuity equation of $\phi$ is

$$
\dot{\rho}_{\phi}+3 H\left(\rho_{\phi}+p_{\phi}\right)=\mathcal{Z} Q
$$

we introduce a parameter $\mathcal{Z}$ to denote the different cases of energy exchange between scalar field and the matter. When $\mathcal{Z}=1$, there is energy exchange. When $\mathcal{Z}=0$, there is no energy exchange.

Eq.(11) lead to the K-G equation

$$
\ddot{\phi}+3 H \dot{\phi}-\lambda \kappa V=\mathcal{Z} \frac{Q}{\dot{\phi}}
$$

Insert Eq.(9,10) to Eq.(1,2), we have

$$
\begin{array}{r}
H^{2}=\frac{\kappa^{2}}{3}\left(\rho_{m}+\frac{\dot{\phi}^{2}}{2}+V\right)+f(t) \\
\dot{H}=-\frac{\kappa^{2}}{2}\left(\gamma \rho_{m}+\dot{\phi}^{2}\right)+g(t)-f(t)
\end{array}
$$

where we use the state equation of matter $p_{m}=w \rho_{m}$ and set $\gamma=1+w . \quad \gamma$ is adiabatic index, it satisfies $0<\gamma<2$. Its value is $4 / 3$ when there is radiation, and is 1 for standard matter or dark matter.

A more general situation of $\gamma$ is $0 \leq \gamma \leq 2$ [47]. If $\gamma=0$ or $\gamma=2$, the eigenvalues corresponding to some fixed points will have zero elements. To analyze the stability of these points, we need more complex methods just as we do in the Appendix. In this paper, we just consider the barotropic fluid which includes radiation and dust, and $\gamma \neq 0,2$.

Eq. $(12,13,14)$ characterize an autonomous system. We will analyze the dynamical behavior of this system in two type models of entropic cosmology. 


\section{III. $\Lambda(t)$ TYPE}

\section{A. Basic equations}

In $\Lambda(t)$ type model of entropic cosmology, $f(t)=g(t)$ [17]. Therefore the Eq. $(12,13,14)$ change to

$$
\begin{aligned}
& H^{2}=\frac{\kappa^{2}}{3}\left(\rho_{m}+\frac{\dot{\phi}^{2}}{2}+V\right)+f(t) \\
& \ddot{\phi}+3 H \dot{\phi}-\lambda \kappa V=-\mathcal{Z} \frac{3}{\kappa^{2} \dot{\phi}} \dot{f}(t) \\
& \dot{H}=-\frac{\kappa^{2}}{2}\left(\gamma \rho_{m}+\dot{\phi}^{2}\right)
\end{aligned}
$$

To rewrite the Eq. $(15,16,17)$, we introduce following dimensionless variables: [48-50]

$$
x^{2}=\frac{\kappa^{2} \dot{\phi}^{2}}{6 H^{2}}, \quad y^{2}=\frac{\kappa^{2} V}{3 H^{2}}, \quad z=\frac{H_{0}}{H_{0}+H}
$$

The variable $z$ ensures that the physical phasespace is compact. The Hubble parameter $H \rightarrow 0$ in the early time universe and $H \rightarrow \infty$ for the late time universe. Therefore [50]

$$
z= \begin{cases}1 & (H \rightarrow 0) \\ 1 / 2 & \left(H=H_{0}\right) \\ 0 & (H \rightarrow \infty)\end{cases}
$$

Using these new variables, one can obtain

$$
\frac{\kappa^{2} \rho_{m}}{3 H^{2}}=1-\alpha_{1}-x^{2}-y^{2}-\alpha_{3} \frac{z}{1-z}-\alpha_{4}\left(\frac{z}{1-z}\right)^{2}
$$

Using the new variables, and considering Eq.(20), one can rewrite Eq. $(15,16,17)$ in the following forms:

$$
\begin{aligned}
\frac{d x}{d N}= & -3 x+\sqrt{\frac{3}{2}} \lambda y^{2}+\frac{3}{2}\left\{\gamma\left[1-\alpha_{1}-x^{2}-y^{2}-\alpha_{3} \frac{z}{1-z}-\alpha_{4}\left(\frac{z}{1-z}\right)^{2}\right]+2 x^{2}\right\} \\
& \times\left\{x+\frac{\mathcal{Z}}{x}\left[\alpha_{1}+\alpha_{3} \frac{z}{2(1-z)}\right]\right\} \\
\frac{d y}{d N}= & -\sqrt{\frac{3}{2} \lambda x y+} \frac{3}{2} y\left\{\gamma\left[1-\alpha_{1}-x^{2}-y^{2}-\alpha_{3} \frac{z}{1-z}-\alpha_{4}\left(\frac{z}{1-z}\right)^{2}\right]+2 x^{2}\right\} \\
\frac{d z}{d N}= & \frac{3}{2} z(1-z)\left\{\gamma\left[1-\alpha_{1}-x^{2}-y^{2}-\alpha_{3} \frac{z}{1-z}-\alpha_{4}\left(\frac{z}{1-z}\right)^{2}\right]+2 x^{2}\right\}
\end{aligned}
$$

where $N \equiv \ln a$.

If we selected the suitable value for $\lambda, \gamma, \alpha_{1}, \alpha_{3}, \alpha_{4}$, the right-hand of Eq. $(21,22,23)$ only depend on $x, y, z$, but not on $N$. Such a dynamical system is usually called an autonomous system. Inspection of the dynamical equations shows that system Eq. $(21,22,23)$ is invariant under the transformation $y \mapsto-y$. This implies that we can restrict our analysis on the half phase space with $y>0$.

We consider two different cases of energy exchange, i.e. $\mathcal{Z}=1$ and $\mathcal{Z}=0$. The fixed point of this autonomous system are shown in Table I for $\mathcal{Z}=1$ and Table II for $\mathcal{Z}=0$.

where

$$
\begin{aligned}
x_{\Lambda_{2}^{ \pm}} & =\frac{\lambda^{2}+3 \gamma \pm \sqrt{\left(\lambda^{2}-3 \gamma\right)^{2}+12 \alpha_{1} \lambda^{2} \gamma}}{2 \sqrt{6} \lambda} \\
y_{\Lambda_{2}^{ \pm}} & =\left[\left(\frac{2}{\gamma}-1\right)\left(x_{\Lambda_{2}^{ \pm}}\right)^{2}-\sqrt{\frac{2}{3}} \frac{\lambda}{\gamma} x_{\Lambda_{2}^{ \pm}}+1-\alpha_{1}\right]^{1 / 2} \\
z_{\Lambda_{3}} & =\frac{2-2 \alpha_{1}+\alpha_{3}-\sqrt{\alpha_{3}^{2}+4 \alpha_{4}-4 \alpha_{1} \alpha_{4}}}{2\left(1-\alpha_{1}+\alpha_{3}-\alpha_{4}\right)}
\end{aligned}
$$

There are some remarks on Table I and II:
TABLE I. Fixed point for $\mathcal{Z}=1$ in $\Lambda(t)$-type

\begin{tabular}{ccccc}
\hline \hline Fixed point & $x_{f}$ & $y_{f}$ & $z_{f}$ & Existence \\
\hline$\Gamma_{1}^{+}$ & $\sqrt{\frac{\alpha_{1} \gamma}{2-\gamma}}$ & 0 & 0 & $\forall \lambda$ and $\gamma$ \\
$\Gamma_{1}^{-}$ & $-\sqrt{\frac{\alpha_{1} \gamma}{2-\gamma}}$ & 0 & 0 & $\forall \lambda$ and $\gamma$ \\
$\Gamma_{2}^{+}$ & $\sqrt{1-\alpha_{1}}$ & 0 & 0 & $0 \leq \alpha_{1} \leq 1$ \\
$\Gamma_{2}^{-}$ & $-\sqrt{1-\alpha_{1}}$ & 0 & 0 & $0 \leq \alpha_{1} \leq 1$ \\
$\Gamma_{3}$ & $\lambda / \sqrt{6}$ & $\sqrt{1-\alpha_{1}-\frac{\lambda^{2}}{6}}$ & 0 & $\lambda^{2}<6\left(1-\alpha_{1}\right)$ \\
$\Gamma_{4}$ & $\sqrt{\frac{3}{2}} \frac{\gamma}{\lambda}$ & $\sqrt{\frac{3(2-\gamma) \gamma-2 \alpha_{1} \lambda^{2}}{2 \lambda^{2}}}$ & 0 & $\lambda^{2}>3 \gamma$ \\
\hline \hline
\end{tabular}

- When $\mathcal{Z}=1$, the fixed points are all in $x-y$ plane. If the value of $\lambda$ and $\gamma$ have been selected, the position of fixed points depend on $\alpha_{1}$ only. In other words, the value of $\alpha_{3}$ and $\alpha_{4}$ dose not affect the position of fixed points.

- When $\mathcal{Z}=0$, the fixed points are in $x-y$ plane except $\Lambda_{3}$. The positon of all the fixed points in $x-y$ plane depend only on $\alpha_{1}$ like in $\mathcal{Z}=1$ case. 
TABLE II. Fixed point for $\mathcal{Z}=0$ in $\Lambda(t)$-type

\begin{tabular}{ccccc}
\hline \hline Fixed point & $x_{f}$ & $y_{f}$ & $z_{f}$ & Existence \\
\hline$\Lambda_{0}$ & 0 & 0 & 0 & $\forall \lambda$ and $\gamma$ \\
$\Lambda_{1}^{+}$ & $\sqrt{1+\frac{\alpha_{1} \gamma}{2-\gamma}}$ & 0 & 0 & $\forall \lambda$ and $\gamma$ \\
$\Lambda_{1}^{-}$ & $-\sqrt{1+\frac{\alpha_{1} \gamma}{2-\gamma}}$ & 0 & 0 & $\forall \lambda$ and $\gamma$ \\
$\Lambda_{2}^{+}$ & $x_{\Lambda_{2}^{+}}$ & $y_{\Lambda_{2}^{+}}$ & 0 & $(\lambda, \gamma) \in\left\{y_{\Lambda_{2}^{+}} \geq 0\right\}$ \\
$\Lambda_{2}^{-}$ & $x_{\Lambda_{2}^{-}}$ & $y_{\Lambda_{2}^{-}}$ & 0 & $(\lambda, \gamma) \in\left\{y_{\Lambda_{2}^{-}} \geq 0\right\}$ \\
$\Lambda_{3}$ & 0 & 0 & $z_{\Lambda_{3}}$ & $\forall \lambda$ and $\gamma$ \\
\hline \hline
\end{tabular}

The influences of $\alpha_{3}$ and $\alpha_{4}$ on fixed points is only reflected in the position of $\Lambda_{3}$.

- For C-version $\left(\alpha_{1}=\alpha_{3}=0\right)$ and H-version $\left(\alpha_{1}=\right.$ $\left.\alpha_{4}=0\right)$ of $\Lambda(t)$-type, $\Gamma_{1}^{ \pm}$are origin of phase space, and $\Gamma_{2}^{ \pm}, \Gamma_{3}, \Gamma_{4}$ are same as in normal cosmology. It means that, when $\mathcal{Z}=1$ the appearance of $H$ and constant terms does not affect the position of fixed points.

- $\Lambda_{3}$ is $\left(0,0, \frac{1}{1+\sqrt{\alpha_{4}}}\right)$ for C-version and is $\left(0,0, \frac{1}{1+\alpha_{3}}\right)$ for H-version.

\section{B. Stability of fixed points}

For simplicity, we define $\frac{d x}{d N}=F_{1}(x, y, z), \frac{d y}{d N}=$ $F_{2}(x, y, z)$ and $\frac{d z}{d N}=F_{3}(x, y, z)$. The properties of each fixed points are determined by the eigenvalues of the Jacobian matrix $[51,52]$

$$
J=\left.\left(\begin{array}{lll}
\frac{\partial F_{1}}{\partial x} & \frac{\partial F_{1}}{\partial y} & \frac{\partial F_{1}}{\partial z} \\
\frac{\partial F_{2}}{\partial x} & \frac{\partial F_{2}}{\partial y} & \frac{\partial F_{2}}{\partial z} \\
\frac{\partial F_{3}}{\partial x} & \frac{\partial F_{3}}{\partial y} & \frac{\partial F_{3}}{\partial z}
\end{array}\right)\right|_{\left(x_{f}, y_{f}, z_{f}\right)}
$$

We will discuss the eigenvalues of $J$ in two different cases of energy exchange, i.e. $\mathcal{Z}=1$ and $\mathcal{Z}=0$.

$$
\text { 1. } \mathcal{Z}=1
$$
III.

Eigenvalues of $J$ for $\mathcal{Z}=1$ in $\Lambda(t)$ type shown in Table where

$$
\begin{aligned}
\alpha_{1}^{*}= & 1-\alpha_{1} \\
\mathcal{M}= & -3 \lambda \gamma(2-\gamma)-2 \alpha_{1} \lambda\left(\lambda^{2}-6 \gamma\right) \\
\mathcal{N}= & \left\{9(2-\gamma) \gamma^{2}\left[\lambda^{2}(2-9 \lambda)+24 \gamma^{2}\right]+4 \alpha_{1}^{2} \lambda^{2}\left(\lambda^{2}-6 \gamma\right)^{2}\right. \\
& \left.+12 \alpha_{1} \lambda^{2} \gamma\left[\lambda^{2}(2+3 \gamma)-6 \lambda(2+\gamma)\right]\right\}^{1 / 2} \\
= & \sqrt{\mathcal{M}^{2}+\mathcal{R}}
\end{aligned}
$$

and

$$
\begin{aligned}
\mathcal{R}= & 9(2-\gamma) \gamma^{2}\left(\lambda^{2} \gamma+24 \gamma^{2}-9 \lambda^{3}\right)+24 \alpha_{1} \lambda^{2} \gamma\left[2 \lambda^{2} \gamma\right. \\
& +3 \lambda(2+\gamma)+3 \gamma(2-\gamma)]
\end{aligned}
$$

TABLE III. Eigenvalues of $J$ for $\mathcal{Z}=1$ in $\Lambda(t)$-type

\begin{tabular}{cc}
\hline \hline Fixed point & Eigenvalues \\
\hline$\Gamma_{1}^{+}$ & $\left\{\frac{3 \gamma}{2}, \quad \frac{1}{2}\left(3 \gamma-\sqrt{6} \lambda \sqrt{\frac{\alpha_{1}}{2-\gamma}}\right), 3 \gamma-6 \alpha_{1}^{*}\right\}$ \\
$\Gamma_{1}^{-}$ & $\left\{\frac{3 \gamma}{2}, \quad \frac{1}{2}\left(3 \gamma+\sqrt{6} \lambda \sqrt{\frac{\alpha_{1}}{2-\gamma}}\right), 3 \gamma-6 \alpha_{1}^{*}\right\}$ \\
$\Gamma_{2}^{+}$ & $\left\{3 \alpha_{1}^{*}, 3 \alpha_{1}^{*}-\sqrt{\frac{3}{2}} \sqrt{\alpha_{1}^{*}} \lambda, 6 \alpha_{1}^{*}-3 \gamma\right\}$ \\
$\Gamma_{2}^{-}$ & $\left\{3 \alpha_{1}^{*}, 3 \alpha_{1}^{*}+\sqrt{\frac{3}{2}} \sqrt{\alpha_{1}^{*}} \lambda, 6 \alpha_{1}^{*}-3 \gamma\right\}$ \\
$\Gamma_{3}$ & $\left\{\frac{1}{2}\left(\lambda^{2}-6 \alpha_{1}^{*}\right), \lambda^{2}-3 \gamma, \frac{\lambda^{2}}{2}\right\}$ \\
$\Gamma_{4}$ & $\left\{\frac{3 \gamma}{2}, \frac{\mathcal{M}-\mathcal{N}}{4 \lambda \gamma}, \frac{\mathcal{M}+\mathcal{N}}{4 \lambda \gamma}\right\}$ \\
\hline \hline
\end{tabular}

We can conclude that:

(1) If $\lambda^{2} \alpha_{1}>3 / 2$, then $3 \gamma-\sqrt{6} \lambda \sqrt{\frac{\alpha_{1}}{2-\gamma}}<0$ for all $0<$ $\gamma<2$. So $\Gamma_{1}^{+}$is a saddle fixed point, its stability property does not depend on the $\gamma$.

(2) If $\lambda^{2} \alpha_{1}<3 / 2$, then the stabiliy property of $\Gamma_{1}^{+}$ is depend on the value of $\gamma$.It may be noted that, when $1-\sqrt{1-\frac{2}{3} \lambda^{2} \alpha_{1}}<\gamma<1+\sqrt{1-\frac{2}{3} \lambda^{2} \alpha_{1}}$, $3 \gamma-\sqrt{6} \lambda \sqrt{\frac{\alpha_{1}}{2-\gamma}}>0$. On the other hand, when $\gamma>2\left(1-\alpha_{1}\right), 3 \gamma-6 \alpha_{1}^{*}>0$. So, when $\lambda^{2} \alpha_{1}<3 / 2$ and $\gamma \in \mathcal{P}:=\left(1-\sqrt{1-\frac{2}{3} \lambda^{2} \alpha_{1}}, 1+\sqrt{1-\frac{2}{3} \lambda^{2} \alpha_{1}}\right) \cap$ $\left(2 \alpha_{1}^{*}, 2\right), \Gamma_{1}^{+}$is a unstable fixed point. If $0<\alpha_{1}<$ $1 / 2$, then $\mathcal{P}=\left(2 \alpha_{1}^{*}, 1+\sqrt{1-\frac{2}{3} \lambda^{2} \alpha_{1}}\right)$. If $\alpha_{1}>1$, then $\mathcal{P}=\left(1-\sqrt{1-\frac{2}{3} \lambda^{2} \alpha_{1}}, 1+\sqrt{1-\frac{2}{3} \lambda^{2} \alpha_{1}}\right)$.

(3) If $\alpha_{1}>1$, then $3 \gamma-6 \alpha_{1}^{*}$ is always positive. So $\Gamma_{1}^{-}$is always an unstable fixed point. Its stability property does not depend on $\gamma$ and $\lambda$. If, $0<\alpha_{1}<$ 1 , then the stability of $\Gamma_{1}^{-}$is depend on $\gamma$. When $\gamma \in\left(2 \alpha_{1}^{*}, 2\right), \Gamma_{1}^{-}$is unstable fixed point. When $\gamma \in\left(0,2 \alpha_{1}^{*}\right), \Gamma_{1}^{-}$is saddle fixed point. One can notice that, the form of potential (the value of $\lambda$ ) will not affect the stability of $\Gamma_{1}^{-}$.

(4) If $0<\alpha_{1}<1$, and $\lambda^{2}>\max \left[6 \alpha_{1}^{*}, 3 \gamma\right], \Gamma_{3}$ will be a unstable fixed point. Otherwise, $\Gamma_{3}$ will be a saddle fixed point. However, the appearing condition for $\Gamma_{3}$ is $\lambda^{2}<6 \alpha_{1}^{*}$. So if $6 \alpha_{1}^{*}>3 \gamma$, i.e. $\alpha_{1}<1-3 \gamma / 2$, $\Gamma_{3}$ is always a saddle fixed point, and if $6 \alpha_{1}^{*}>3 \gamma$, i.e. $1-3 \gamma / 2<\alpha_{1}<1, \Gamma$ is unstable when $\lambda^{2}>3 \gamma$, and $\Gamma$ is saddle when $\lambda^{2}<3 \gamma$.

(5) If $\alpha_{1}>1$, and $\lambda^{2}>3 \gamma, \Gamma_{3}$ will be a unstable fixed point. Otherwise, $\Gamma_{3}$ will be a saddle fixed point.

(6) If $\mathcal{M}>\mathcal{N}$, then $\Gamma_{4}$ is a unstable fixed point. From Eq.(30) we can see that, $\mathcal{N} \geq 0$. It means that $\mathcal{M}$ should be positive, and $\mathcal{R}$ should be negative. From Fig.1, we can see that, there is no intersection between positive $\mathcal{M}$ zone and negative $\mathcal{R}$ zone. Therefore, $\Gamma_{4}$ is a saddle fixed point always. 
TABLE IV. Eigenvalues of $J$ for $\mathcal{Z}=0$ in $\Lambda(t)$-type

\begin{tabular}{|c|c|}
\hline Fixed point & Eigenvalues \\
\hline$\Lambda_{0}$ & $\left\{\frac{3 \gamma}{2} \alpha_{1}^{*}, \quad \frac{3 \gamma}{2} \alpha_{1}^{*}, \quad \frac{3 \gamma}{2} \alpha_{1}^{*}-3\right\}$ \\
\hline$\Lambda_{1}^{+}$ & $\left\{3,6-3 \alpha_{1}^{*} \gamma, 3-\lambda \sqrt{\frac{3}{2} \frac{2-\gamma+\alpha_{1} \gamma}{2-\gamma}}\right.$ \\
\hline$\Lambda_{1}^{-}$ & $\left\{3,6-3 \alpha_{1}^{*} \gamma, 3+\lambda \sqrt{\frac{3}{2} \frac{2-\gamma+\alpha_{1} \gamma}{2-\gamma}}\right.$ \\
\hline$\Lambda_{2}^{+}\left(\alpha_{1}=0\right)$ & $\left\{\frac{1}{2}\left(\lambda^{2}-6\right), \quad \lambda^{2}-3 \gamma, \quad \lambda^{2} / 2\right\}$ \\
\hline$\Lambda_{2}^{-}\left(\alpha_{1}=0\right)$ & $\left\{\frac{3 \gamma}{2}, \quad \frac{\mathcal{M}_{0}-\mathcal{N}_{0}}{4 \lambda \gamma}, \quad \frac{\mathcal{M}_{0}+\mathcal{N}_{0}}{4 \lambda \gamma}\right\}$ \\
\hline$\Lambda_{3}^{C}$ & $\{0,-3,-3 \gamma\}$ \\
\hline$\Lambda_{3}^{H}$ & $\left\{0,-3, \quad-\frac{3 \gamma}{2}\right\}$ \\
\hline
\end{tabular}

$$
\text { 2. } \mathcal{Z}=0
$$

Eigenvalues of $J$ for $\mathcal{Z}=0$ in $\Lambda(t)$-type shown in Table IV. where we consider the special case of $\alpha_{1}=0$ for $\Lambda_{2}^{ \pm}$ and

$$
\begin{aligned}
\mathcal{M}_{0} & =-3 \gamma \lambda(2-\gamma) \\
\mathcal{N}_{0} & =3 \gamma \sqrt{(2-\gamma)\left[\lambda^{2}(2-9 \lambda)+24 \gamma^{2}\right]}
\end{aligned}
$$

The superscript "C" and " $\mathrm{H}$ " of $\Lambda_{3}$ denote the C-version and $\mathrm{H}$-version. We can conclude that:

(1) For $\gamma \in(0,2), \frac{3 \gamma}{2} \alpha_{1}^{*}-3$ is always negative, therefore the stability of $\Lambda_{0}$ depend only on $\alpha_{1}$. If $\alpha_{1} \in(0,1)$, $\Lambda_{0}$ is a saddle fixed point. If $\alpha_{1}>1, \Lambda_{0}$ is an stable fixed point, it is a late time attractor.

(2) For $\gamma \in(0,2), 6-3 \alpha_{1}^{*} \gamma$ is always positive. If $\lambda^{2}<$ $\frac{6(2-\gamma)}{2-\gamma+\alpha_{1} \gamma}, \Lambda_{1}^{+}$is an unstable fixed point. If $\lambda^{2}>$ $\frac{6(2-\gamma)}{2-\gamma+\alpha_{1} \gamma}, \Lambda_{1}+$ is an saddle fixed point.

(3) $\Lambda_{1}^{-}$is always unstable fixed point, it's stability does not depend on the value of $\lambda, \gamma, \alpha_{1}$.

(4) If $\lambda^{2}>6$, then $\lambda^{2}-3 \gamma$ is always positive and $\Lambda_{2}^{+}$is an unstable fixed point. If $\lambda^{2}<6, \Lambda_{2}^{+}$is a saddle fixed point.

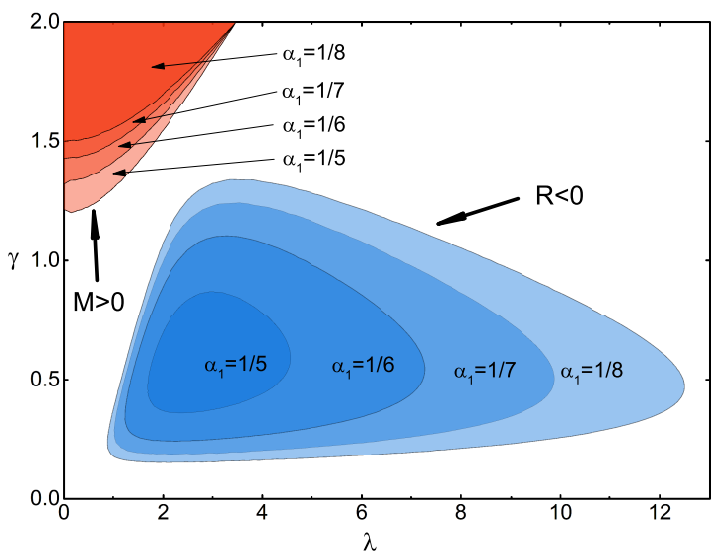

FIG. 1. The parammeter space for $\Gamma_{4}$ is unstable fixed point

(5) $\mathcal{M}_{0}-\mathcal{N}_{0}$ is always negative. Therefore $\Lambda_{2}^{-}$is always a saddle fixed point.

(6) Whether in $\mathrm{C}$-version or $\mathrm{H}$-version, there is an zero eigenvalue for $J$ of $\Lambda_{3}$. It means that $\Lambda_{3}$ is a nonhyperbolic point. Linear stability theory fails for non-hyperbolic points, and the stability property for this point should be determined by centre manifold theory $[51,52]$. We will get the stability property of $\Lambda_{3}$ in Appendix by using centre manifold theory. We can conclude from Appendix that: $\Lambda_{3}$ is a stable fixed point.

From the above discussion, we find that, the stability properties of fixed points depend on the values of the constants $\lambda, \gamma$ and $\alpha_{1}$. It is worth noting that, the values of $\alpha_{3}$ and $\alpha_{4}$ only affect the position of $\Lambda_{3}$. This means the $H^{2}$ terms (area entropy) decide the dynamical properties of the dynamical system Eq. $(21,22,23)$ when $\lambda$ and $\gamma$ have been selected.

Therefore, it can be expected that the dynamical properties in $\mathrm{C}$-version and $\mathrm{H}$-version of entropic cosmology model (where $\alpha_{1}=0$ ) are similar.

\section{BV-TYPE}

In BV type model of entropic cosmology, $f(t)=0$. Therefore, Eq. $(12,13,14)$ change to

$$
\begin{aligned}
& H^{2}=\frac{\kappa^{2}}{3}\left(\rho_{m}+\frac{\dot{\phi}^{2}}{2}+V\right) \\
& \ddot{\phi}+3 H \dot{\phi}-\lambda \kappa V=\mathcal{Z} \frac{6 H}{\kappa^{2} \dot{\phi}} g(t) \\
& \dot{H}=-\frac{\kappa^{2}}{2}\left(\gamma \rho_{m}+\dot{\phi}^{2}\right)+g(t)
\end{aligned}
$$

As in Section III, we introduce dimensionless variables Eq.(18). From Eq.(34), one can obtain

$$
\frac{\kappa^{2} \rho_{m}}{3 H^{2}}=1-x^{2}-y^{2} \geq 0
$$

This lead to $0 \leq x^{2}+y^{2} \leq 1$, which means the phasespace corresponds to a half-cylinder of unit height and unit radius.

Using the new variables and considering Eq.(37), one can rewrite Eq. $(34,35,36)$ into following forms: 


$$
\begin{aligned}
& \frac{d x}{d N}=-3 x+\sqrt{\frac{3}{2}} \lambda y^{2}+\frac{3}{2} x\left[\gamma\left(1-x^{2}-y^{2}\right)+2 x^{2}\right]+\left[\frac{\mathcal{Z}}{x}-x\right]\left[\beta_{1}+\beta_{3} \frac{z}{1-z}+\beta_{4}\left(\frac{z}{1-z}\right)^{2}\right] \\
& \frac{d y}{d N}=-\sqrt{\frac{3}{2}} \lambda x y+\frac{3}{2} y\left[\gamma\left(1-x^{2}-y^{2}\right)+2 x^{2}\right]-y\left[\beta_{1}+\beta_{3} \frac{z}{1-z}+\beta_{4}\left(\frac{z}{1-z}\right)^{2}\right] \\
& \frac{d z}{d N}=z(1-z)\left\{\frac{3}{2}\left[\gamma\left(1-x^{2}-y^{2}\right)+2 x^{2}\right]-\left[\beta_{1}+\beta_{3} \frac{z}{1-z}+\beta_{4}\left(\frac{z}{1-z}\right)^{2}\right]\right\}
\end{aligned}
$$

TABLE V. Fixed point for $\mathcal{Z}=1$ in BV-type

\begin{tabular}{ccccc}
\hline \hline Fixed point & $x_{f}$ & $y_{f}$ & $z_{f}$ & Existence \\
\hline$\Omega_{1}^{+}$ & 1 & 0 & 0 & $\forall \lambda$ and $\gamma$ \\
$\Omega_{1}^{-}$ & -1 & 0 & 0 & $\forall \lambda$ and $\gamma$ \\
$\Omega_{2}$ & $\lambda / \sqrt{6}$ & $\sqrt{1-\frac{\lambda^{2}}{6}}$ & 0 & $\lambda^{2}<6$ \\
$\Omega_{3}$ & $\sqrt{\frac{3}{2}} \frac{\gamma}{\lambda}$ & $\sqrt{\frac{3(2-\gamma) \gamma}{2 \lambda^{2}}}$ & 0 & $\lambda^{2}>3 \gamma$ \\
$\Omega_{4}^{+}$ & 1 & 0 & $z_{\Omega_{4}}$ & $\forall \lambda$ and $\gamma$ \\
$\Omega_{4}^{-}$ & -1 & 0 & $z_{\Omega_{4}}$ & $\forall \lambda$ and $\gamma$ \\
\hline \hline
\end{tabular}

TABLE VI. Fixed point for $\mathcal{Z}=0$ in BV-type

\begin{tabular}{ccccc}
\hline Fixed point & $x_{f}$ & $y_{f}$ & $z_{f}$ & Existence \\
\hline$\Pi_{0}$ & 0 & 0 & 0 & $\forall \lambda$ and $\gamma$ \\
$\Pi_{1}^{+}$ & 1 & 0 & 0 & $\forall \lambda$ and $\gamma$ \\
$\Pi_{1}^{-}$ & -1 & 0 & 0 & $\forall \lambda$ and $\gamma$ \\
$\Pi_{2}$ & $\lambda / \sqrt{6}$ & $\sqrt{1-\frac{\lambda^{2}}{6}}$ & 0 & $\lambda^{2}<6$ \\
$\Pi_{3}$ & $\sqrt{\frac{3}{2}} \frac{\gamma}{\lambda}$ & $\sqrt{\frac{3(2-\gamma) \gamma}{2 \lambda^{2}}}$ & 0 & $\lambda^{2}>3 \gamma$ \\
$\Pi_{4}$ & 0 & 0 & $z_{\Pi_{4}}$ & $\forall \lambda$ and $\gamma$ \\
\hline \hline
\end{tabular}

Eq. $(38,39,40)$ form an autonomous system.

To discuss the $H$ terms and the constant terms in dynamical system, we consider the $\mathrm{H}$-version and $\mathrm{C}$-version of the entropic cosmology, i.e. we set $\beta_{1}=0$.

The fixed points of this autonomous system shown in Table $\mathrm{V}$ for $\mathcal{Z}=1$ and Table VI for $\mathcal{Z}=0$. where

$$
z_{\Omega_{4}}= \begin{cases}\frac{3}{3+\sqrt{3 \beta_{4}}} & \text { C-version } \\ \frac{3}{3+\beta_{3}} & \text { H-version }\end{cases}
$$

and

$$
z_{\Pi_{4}}= \begin{cases}\frac{3 \gamma}{3 \gamma+\sqrt{6 \gamma \beta_{4}}} & \text { C-version } \\ \frac{3 \gamma}{3 \gamma+2 \beta_{3}} & \text { H-version }\end{cases}
$$

The eigenvalues of Jacobian matrix $J$ are shown in Table VII where

$$
\sigma=\left\{\begin{array}{ll}
6 & \text { C-version } \\
3 & \text { H-version }
\end{array} \text { and } \theta= \begin{cases}3 \gamma & \text { C-version } \\
3 \gamma / 2 & \text { H-version }\end{cases}\right.
$$

We can conclude that:
TABLE VII. Eigenvalues of $J$ for BV-type

\begin{tabular}{cc}
\hline \hline Fixed point(s) & Eigenvalues \\
\hline$\Pi_{0}$ & $\left\{\frac{3}{2}(\gamma-2), \frac{3}{2} \gamma, \frac{3}{2} \gamma\right\}$ \\
$\Omega_{1}^{ \pm}, \Pi_{1}^{ \pm}$ & $\left\{3(2-\gamma), 3 \mp \sqrt{\frac{3}{2}} \lambda, 3\right\}$ \\
$\Omega_{2}, \Pi_{2}$ & $\left\{\lambda^{2} / 2,\left(\lambda^{2}-6\right) / 2, \lambda^{2}-3 \gamma\right\}$ \\
$\Omega_{3}, \Pi_{3}$ & $\left\{\frac{3 \gamma}{2}, \frac{\mathcal{M}_{0}-\mathcal{N}_{0}}{4 \lambda \gamma}, \frac{\mathcal{M}_{0}+\mathcal{N}_{0}}{4 \lambda \gamma}\right\}$ \\
$\Omega_{4}^{ \pm}$ & $\left\{\begin{array}{c}\left.-\sigma,-3 \gamma, \mp \sqrt{\frac{3}{2}} \lambda\right\} \\
\Pi_{4}\end{array}\right.$ \\
\hline \hline
\end{tabular}

(1) For $\gamma \in(0,2), \Pi_{0}$ is always an saddle fixed point. The stability properties of the $\Pi_{0}$ is same as $\Lambda_{0}$ when $\alpha_{1}=0$.

(2) For $\gamma \in(0,2), \Omega_{1}^{-}$and $\Pi_{1}^{-}$are always unstable fixed point.

(3) If $\lambda^{2}<6$, then $3-\sqrt{\frac{3}{2}} \lambda>0$. It means that $\Omega_{1}^{+}$ and $\Pi_{1}^{+}$are unstable fixed point. If $\lambda^{2}>6$, then $\Omega_{1}^{+}$and $\Pi_{1}^{+}$are saddle fixed point.

(4) The existence of $\Omega_{2}$ and $\Pi_{2}$ is $\lambda^{2}<6$, which means that $\Omega_{2}$ and $\Pi_{2}$ are saddle fixed point.

(5) For $\gamma \in(0,2), \Omega_{3}$ and $\Pi_{3}$ are saddle point.

(6) $\Omega_{4}^{+}$is always a stable fixed point, and $\Omega_{4}^{-}$is always a saddle fixed point.

(7) $\Pi_{4}$ is a non-hyperbolic point, its stability property is determined by centre manifold theory. We discuss the stability of $\Pi_{4}$ in Appendix, and we can conclude that $\Pi_{4}$ is a stable fixed point.

From the discussion of this section, we can see that, the dynamical structure of $\mathrm{H}$-version and $\mathrm{C}$-version are same. It mean that, $H$ terms and the constant terms have the same influence on dynamical properties.

\section{SUMMARY AND DISCUSSION}

In this paper, we discuss the stability properties of autonomous system in entropic cosmology. It can be found 
that, different entropic terms have different effect on the stability properties of fixed points.

The $H^{2}$ terms do not describe cosmological fluctuation and is neglected in some discussions[17]. However, the influences of $H^{2}$ terms on dynamical behavior of $\phi$ are important.

From the discussion of $\Lambda(t)$ type in Sec.II, one can find that, $\alpha_{3}$ and $\alpha_{4}$ only affect the position of fixed point $\Lambda_{3}$ (see Eq.26). Furthermore, the stability property of $\Lambda_{3}$ is decided by $\alpha_{1}$. If we neglect the $H^{2}$ term, i.e. $\alpha_{1}=0$, the fixed point $\Lambda_{3}$ is always a stable point, its stability property has nothing to do with $H$ term and constant term.

Therefore, it is not surprising that the eigenvalues of $J$ in C-version and $\mathrm{H}$-version of BV type model are independent with $\beta_{3}$ and $\beta_{4}$ (see VII). $H$ terms and constant terms also have no influence on stability property of fixed points.

On the other hand, from the discussion of BV type model in Sec.III, we can see that there is no essential difference between dynamical properties of $\mathrm{H}$-version and $\mathrm{C}$-version. The only difference between two versions of model is the position of fixed points $\Omega_{4}^{ \pm}$and $\Pi_{4}$.

In summary, from the perspective of dynamics, the entropic-force terms can be divided into two classes. The first class of entropic-force terms is proportional to $H^{2}$ (area entropy), they affect the dynamical properties of autonomous system in entropic cosmology. The second class is the linear combination of $H$ terms (volume entropy) and constant terms, they only affect the position of fixed points, they have no influences on dynamical structure.

\section{ACKNOWLEDGMENTS}

This work was supported by the National Natural Science Foundation of China under Grant No.11447198 (Fund of theoretical physics), National Natural Science Foundation of China under Grant No.11305021 and the Fundamental Research Funds for the Central Universities of Ministry of Education of China under Grants No. 3132015234

\section{Appendix A: The stability properties of the non-hyperbolic points $\Lambda_{3}$ and $\Pi_{4}$}

As we have point out in Section III and IV, if the eigenvalues of the Jacobi matrix had one or more eigenvalues with zero real parts while the rest of the eigenvalues had negative real parts, then linearization failed to determine the stability properties of this critical point [51, 52]. $\Lambda_{3}$ and $\Pi_{4}$ are such points. In this Appendix, we determine the stability properties of these points by using centre manifold theory [52].
1. $\Lambda_{3}$

We transfer $\Lambda_{3}$ to $\Lambda_{3}^{\prime}\left(x_{f}=0, y_{f}=0, z_{f}^{*}=z_{f}-z_{\Lambda_{3}}=\right.$ $0)$ for convenience at first, where

$$
z_{\Lambda_{3}}= \begin{cases}\frac{1}{\sqrt{\alpha_{4}}+1} & \text { C-version } \\ \frac{1}{\alpha_{3}+1} & \text { H-version }\end{cases}
$$

Then Eq. $(21,22,23)$ can be rewritten as

$$
\begin{aligned}
\frac{d x}{d N}= & \frac{3}{2} x\left[\gamma\left(1-x^{2}-y^{2}-\mathcal{T}\left(z^{*}\right)\right)+2 x^{2}\right] \\
& -3 x+\sqrt{\frac{3}{2}} \lambda y^{2} \\
\frac{d y}{d N}= & \frac{3}{2} y\left[\gamma\left(1-x^{2}-y^{2}-\mathcal{T}\left(z^{*}\right)\right)+2 x^{2}\right] \\
& -\sqrt{\frac{3}{2}} \lambda x y \\
\frac{d z^{*}}{d N}= & \frac{3}{2}\left[\gamma\left(1-x^{2}-y^{2}-\mathcal{T}\left(z^{*}\right)\right)+2 x^{2}\right] \\
& \times\left(z^{*}+z_{\Lambda_{3}}\right)\left(1-z^{*}-z_{\Lambda_{3}}\right)
\end{aligned}
$$

where

$$
\mathcal{T}\left(z^{*}\right)= \begin{cases}\alpha_{4}\left(\frac{z^{*}+z_{\Lambda_{3}}}{1-z^{*}-z_{\Lambda_{3}}}\right)^{2} & \text { C-version } \\ \alpha_{3} \frac{z^{*}+z_{\Lambda_{3}}}{1-z^{*}-z_{\Lambda_{3}}} & \text { H-version }\end{cases}
$$

The Jacobi matrix $J$ of dynamical system Eq.(A2,A3,A4) at point $\Lambda_{3}^{\prime}$ is

$$
J=\left(\begin{array}{ccc}
-3 & 0 & 0 \\
0 & 0 & 0 \\
0 & 0 & -\theta
\end{array}\right)
$$

where

$$
\theta= \begin{cases}3 \gamma & \text { C-version } \\ 3 \gamma / 2 & \text { H-version }\end{cases}
$$

The eigenvectors for eigenvalues $(-3,0,-\theta)$ are

$$
(1,0,0)^{T}, \quad(0,1,0)^{T}, \quad(0,0,1)^{T}
$$

respectively.

The stable subspaces $\mathcal{E}^{s}$ is spanned by the eigenvectors of $J$ which have negative real part. In the case of our discussion, $\mathcal{E}^{s}$ is $x-z^{*}$ plane. The centre subspaces $\mathcal{E}^{c}$ is spanned by the eigenvectors of $J$ which have zero real part. In our case, $\mathcal{E}^{c}$ is $y$ axis. Linear stability theory is sufficient to understand the dynamics of trajectories in $\mathcal{E}^{s}$, and the dynamics of trajectories in $\mathcal{E}^{c}$ is determined by centre manifold theory [52].

Therefore, we set centre manifold $W^{c}$ is

$$
W^{c}\left(\Lambda_{3}^{\prime}\right)=\left\{\left(h_{1}(y), y, h_{2}(y)\right): y \in[0,=\infty)\right\}
$$

where

$$
h_{1}(0)=h_{2}(0)=\nabla h_{1}(0)=\nabla h_{2}(0)=0
$$


Therefore, we can assume that

$$
\begin{aligned}
& h_{1}(y)=a_{1} y^{2}+a_{2} y^{3}+\cdots \\
& h_{2}(y)=a_{3} y^{2}+a_{4} y^{3}+\cdots
\end{aligned}
$$

The vector $\left(\frac{d x}{d N}, \frac{d y}{d N}, \frac{d z}{d N}\right)$ on a trajectory in $W^{c}$ must tangent to $W^{c}$. This means

$$
x(N)=h_{1}(y(N)), \quad z^{*}(N)=h_{2}(y(N))
$$

i.e.

$$
\frac{d x}{d N}=\nabla h_{1}(y) \frac{d y}{d N}, \quad \frac{d z^{*}}{d N}=\nabla h_{2}(y) \frac{d y}{d N}
$$

Substitute Eq.(A2,A3,A4) in Eq.(A14) and using Eq.(A11,A12):

$$
\begin{aligned}
& -3 x+\sqrt{\frac{3}{2}} \lambda y^{2}+\frac{3}{2} x\left[\gamma\left(1-x^{2}-y^{2}-\mathcal{R}\left(z^{*}\right)\right)+2 x^{2}\right] \\
= & \left(2 a_{1} y+3 a_{2} y^{2}\right)\left\{-\sqrt{\frac{3}{2}} \lambda x y+\frac{3}{2} y\left[\gamma\left(1-x^{2}-y^{2}-\mathcal{R}\left(z^{*}\right)\right)+2 x^{2}\right]\right\} \\
& \frac{3}{2}\left[\gamma\left(1-x^{2}-y^{2}-\mathcal{R}\left(z^{*}\right)\right)+2 x^{2}\right]\left(z^{*}+z_{\Lambda_{3}}\right)\left(1-z^{*}-z_{\Lambda_{3}}\right) \\
= & \left(2 a_{3} y+3 a_{4} y^{2}\right)\left\{-\sqrt{\frac{3}{2}} \lambda x y+\frac{3}{2} y\left[\gamma\left(1-x^{2}-y^{2}-\mathcal{R}\left(z^{*}\right)\right)+2 x^{2}\right]\right\}
\end{aligned}
$$

Substitute $x=h_{1}(y), z^{*}=h_{2}(y)$ into Eq.(A15,A16) and notice that the coefficients of each power of $y$ must be zero, we can solve that

$$
a_{1}=\frac{\lambda}{\sqrt{6}}, a_{2}=a_{4}=0, a_{3}= \begin{cases}\frac{\sqrt{\alpha_{4}}}{2\left(1+\sqrt{\alpha_{4}}\right)^{2}} & \text { C-version } \\ -\frac{\alpha_{3}}{\left(1+\alpha_{3}\right)^{2}} & \text { H-version }\end{cases}
$$

Therefore the dynamics equation for $y$ is

$$
\frac{d y}{d N}=-\frac{\lambda^{2}}{2} y^{3}+O\left(y^{4}\right)
$$

We conclude that, for $y$ sufficiently small, $y=0$ is stable, therefore $\Lambda_{3}$ is an stable fixed point.

\section{2. $\Pi_{4}$}

The discussion of the $\Pi_{4}$ is same as $\Lambda_{3}$. We transfer $\Pi_{4}$ to $\Pi_{4}^{\prime}\left(x_{f}=0, y_{f}=0, z_{f}^{*}=z_{f}-z_{\Pi_{4}}=0\right)$. Then the Eq. $(38,39,40)$ change to

$$
\begin{aligned}
\frac{d x}{d N}= & \frac{3}{2} x\left[\gamma\left(1-x^{2}-y^{2}\right)+2 x^{2}\right]-x \mathcal{S}\left(z^{*}\right) \\
& -3 x+\sqrt{\frac{3}{2}} \lambda y^{2} \\
\frac{d y}{d N}= & \frac{3}{2} y\left[\gamma\left(1-x^{2}-y^{2}\right)+2 x^{2}\right]-y \mathcal{S}\left(z^{*}\right) \\
& -\sqrt{\frac{3}{2}} \lambda x y \\
\frac{d z^{*}}{d N}= & \left\{\frac{3}{2}\left[\gamma\left(1-x^{2}-y^{2}\right)+2 x^{2}\right]-\mathcal{S}\left(z^{*}\right)\right\} \\
& \times\left(z^{*}+z_{\Pi_{4}}\right)\left(1-z^{*}-z_{\Pi_{4}}\right)
\end{aligned}
$$

where

$$
\mathcal{S}\left(z^{*}\right)=\left\{\begin{array}{lll}
\beta_{4}\left(\frac{z^{*}+z_{\Pi_{4}}}{1-z^{*}-z_{\Pi_{4}}}\right)^{2} & \text { C-version } & \text { Eq.(A26,A27): } \\
\beta_{3} \frac{z^{*}+z_{\Pi_{4}}}{1-z^{*}-z_{\Pi_{4}}} & \text { H-version } & \text { https://mc06.manuscriptcentral.com/cjp-pubs }
\end{array}\right.
$$

The Jacobi matrix $J$ of dynamical system Eq. $(A 18, A 19, A 20)$ at point $\Pi_{4}^{\prime}$ is

$$
J=\left(\begin{array}{ccc}
-3 & 0 & 0 \\
0 & 0 & 0 \\
0 & 0 & -\theta
\end{array}\right)
$$

The eigenvectors for eigenvalues $(-3,0,-\theta)$ are

$$
(1,0,0)^{T}, \quad(0,1,0)^{T}, \quad(0,0,1)^{T}
$$

respectively.

We set centre manifold $W^{c}$ is

$$
W^{c}\left(\Lambda_{3}^{\prime}\right)=\left\{\left(h_{1}^{\prime}(y), y, h_{2}^{\prime}(y)\right): y \in[0,=\infty)\right\}
$$

where

$$
h_{1}^{\prime}(0)=h_{2}^{\prime}(0)=\nabla h_{1}^{\prime}(0)=\nabla h_{2}^{\prime}(0)=0
$$

Therefore, we can assume that

$$
\begin{aligned}
& h_{1}^{\prime}(y)=b_{1} y^{2}+b_{2} y^{3}+\cdots \\
& h_{2}^{\prime}(y)=b_{3} y^{2}+b_{4} y^{3}+\cdots
\end{aligned}
$$

The vector $\left(\frac{d x}{d N}, \frac{d y}{d N}, \frac{d z^{*}}{d N}\right)$ on a trajectory in $W^{c}$ must tangent to $W^{c}$. This means

$$
x(N)=h_{1}^{\prime}(y(N)), \quad z^{*}(N)=h_{2}^{\prime}(y(N))
$$

i.e.

$$
\frac{d x}{d N}=\nabla h_{1}^{\prime}(y) \frac{d y}{d N}, \quad \frac{d z^{*}}{d N}=\nabla h_{2}^{\prime}(y) \frac{d y}{d N}
$$

Substitute Eq.(A18,A19,A20) in Eq.(A29) and using 


$$
\begin{aligned}
& \frac{3}{2} x\left[\gamma\left(1-x^{2}-y^{2}\right)+2 x^{2}\right]-x \mathcal{S}\left(z^{*}\right)-3 x+\sqrt{\frac{3}{2}} \lambda y^{2} \\
= & \left(2 b_{1} y+3 b_{2} y^{2}\right)\left\{\frac{3}{2} y\left[\gamma\left(1-x^{2}-y^{2}\right)+2 x^{2}\right]-y \mathcal{S}\left(z^{*}\right)-\sqrt{\frac{3}{2}} \lambda x y\right\} \\
& \left\{\frac{3}{2}\left[\gamma\left(1-x^{2}-y^{2}\right)+2 x^{2}\right]-\mathcal{S}\left(z^{*}\right)\right\}\left(z^{*}+z_{\Pi_{4}}\right)\left(1-z^{*}-z_{\Pi_{4}}\right) \\
= & \left(2 b_{3} y+3 b_{4} y^{2}\right)\left\{\frac{3}{2} y\left[\gamma\left(1-x^{2}-y^{2}\right)+2 x^{2}\right]-y \mathcal{S}\left(z^{*}\right)-\sqrt{\frac{3}{2}} \lambda x y\right\}
\end{aligned}
$$

Substitute $x=h_{1}^{\prime}(y), z^{*}=h_{2}^{\prime}(y)$ into Eq.(A30,A31) and notice that the coefficients of each power of $y$ must be zero, we can solve that

$b_{1}=\frac{\lambda}{\sqrt{6}}, b_{2}=b_{4}=0, b_{3}= \begin{cases}\frac{3 \gamma \sqrt{6 \gamma \beta_{4}}}{2\left(3 \gamma+\sqrt{6 \gamma \beta_{4}}\right)^{2}} & \text { C-version } \\ -\frac{6 \beta_{3} \gamma}{\left(3 \gamma+2 \beta_{3}\right)^{2}} & \text { H-version }\end{cases}$
Therefore the dynamics equation for $y$ is

$$
\frac{d y}{d N}=-\frac{\lambda^{2}}{2} y^{3}+O\left(y^{4}\right)
$$

We conclude that, for $y$ sufficiently small, $y=0$ is stable, therefore $\Pi_{4}$ is an stable fixed point.
[1] S. Perlmutter and et al., Nature 391, 51 (1998).

[2] S. Perlmutter and et al., Astrophys. J 517, 565 (1999).

[3] A. G. Riess and et al., Astron. J. 116, 1009 (1998).

[4] A. G. Riess and et al., Astrophys. J. 607, 665 (2004).

[5] A. G. Riess and et al., Astrophys. J. 659, 98 (2007).

[6] S. Weinberg, Cosmology (Oxford University Press, 2008).

[7] P. J. E. Peebles and B. Ratra, Rev. Mod. Phys. 75, 559 (2003).

[8] E. J. Copeland, M. Sami, and S. Tsujikawa, Int. J. Mod. Phys. D. 15, 1753 (2006).

[9] D. A. Easson, P. H. Frampton, and G. F. Smoot, Phys. Lett. B 696, 273 (2011).

[10] D. A. Easson, P. H. Frampton, and G. F. Smoot, Int. J. Mod. Phys. A 27, 1250066 (2012).

[11] E. Verlinde, JHEP 04, 029 (2011).

[12] T. S. Koivisto, D. F. Mota, and M. Zumalacárregui, J. Cosmol. Astropart. Phys. 02, 027 (2011).

[13] S. Basilakos, D. Polarski, and J. Solà, Phys. Rev. D 86, 043010 (2012).

[14] R. Casadio and A. Gruppuso, Phys. Rev. D 84, 023503 (2011).

[15] N. Komatsu and S. Kimura, Phys. Rev. D 88, 083534 (2013).

[16] S. Basilakos and J. Solà, Mon. Not. R. Astron. Soc. 437, 3331 (2014).

[17] N. Komatsu and S. Kimura, Phys. Rev. D 89, 123501 (2014).

[18] K. Freese, F. C. Adams, J. A. Frieman, and E. Mottola, Nuclear Physics B287, 797 (1987).

[19] J. M. Overduin and F. I. Cooperstock, Phys. Rev. D 58, 043506 (1998).

[20] I. L. Shapiro and J. Solà, JHEP 02, 006 (2002).

[21] C. España-Bonet, P. Ruiz-Lapuente, I. L. Shapiro, and J. Solà, J. Cosmo. Astro. Phys. 02, 006 (2004).

[22] S. Carneiro, C. Pigozzo, H. A. Borges, and J. S. Alcaniz, Phys. Rev. D 74, 023532 (2006).

[23] S. Basilakos, M. Plionis, and J. Solà, Phys. Rev. D 80, 083511 (2009).
[24] J. Alcaniz, H. Borges, S. Carneiro, J. Fabrisc, C. Pigozzo, and W. Zimdahl, Phys. Lett. B 716, 165 (2012).

[25] E. L. D. Perico, J. A. S. Lima, S. Basilakos, and J. Solà, Phys. Rev. D 88, 063531 (2013).

[26] G. L. Murphy, Phys. Rev. D 8, 12 (1973).

[27] J. D. Barrow, Phys. Lett. B 180, 4 (1986).

[28] J. A. S. Lima, R. Portugal, and I. Waga, Phys. Rev. D 37, 2755 (1988).

[29] J. A. S. Lima, A. S. M. Germano, and L. R. W. Abramo, Phys. Rev. D 53, 4287 (1996).

[30] W. Zimdahl, D. J. Schwarz, A. B. Balakin, and D. Pavón, Phys. Rev. D 64, 063501 (2001).

[31] S. Nojiri and S. D. Odintsov, Phys. Rev. D 72, 023003 (2005).

[32] S. Capozziello, V. F. Cardone, E. Elizalde, S. Nojiri, and S. D. Odintsov, Phys. Rev. D 73, 043512 (2006).

[33] J. Ren and X.-H. Meng, Phys. Lett. B 633, 1 (2006).

[34] J. A. S. Lima, F. E. Silva, and R. C. Santos, Class. Quantum Grav. 25, 205006 (2008).

[35] W. Hipólito-Ricaldi, H. Velten, and W. Zimdahl, J. Cosmo. Astro. Phys. 06, 016 (2009).

[36] B. Li and J. D. Barrow, Phys. Rev. D 79, 103521 (2009).

[37] S. Basilakos, M. Plionis, and J. A. S. Lima, Phys. Rev. D 82, 083517 (2010).

[38] J. Lima, J. Jesus, and F. Oliveira, J. Cosmo. Astro. Phys. 11, 027 (2010).

[39] I. Brevik, E. Elizalde, S. Nojiri, and S. D. Odintsov, Phys. Rev. D 84, 103508 (2011).

[40] O. F. Piattella, J. C. Fabris, and W. Zimdahl, J. Cosmo. Astro. Phys. 05, 029 (2011).

[41] J. A. S. Lima, S. Basilakos, and F. E. M. Costa, Phys. Rev. D 86, 103534 (2012).

[42] N. Komatsu and S. Kimura, Phys. Rev. D 87, 043531 (2013).

[43] J. D. Barrow and T. Clifton, Phys. Rev. D 73, 103520 (2006).

[44] P. K. Townsend, JHEP 11, 042 (2001).

[45] A. A. Coley and R. J. van den Hooge, Phys. Rev. D 62, 
$023517(2000)$.

[46] F. Finelli, Phys. Lett. B 545, 1 (2002).

[47] A. P. Billyard, A. A. Coley, and R. J. van den Hoogen, Phys. Rev. D 58, 123501 (1998).

[48] E. J. Copeland, A. R. Liddle, and D. Wands, Phys. Rev. D 57, 4686 (1998).

[49] D. Samart and B. Gumjudpai, Phys. Rev. D 76, 043514 (2007).

[50] C. G. Böhmer, G. Caldera-Cabral, R. Lazkoz, and R. Maartens, Phys. Rev. D 78, 023505 (2008).
[51] S. L. Campbell and R. Haberman, Introduction to Differential Equations with Dynamical Systems (Prienceton University Press, 2008).

[52] J. D. Meiss, Differential Dynamical Systems (Society for Industrial and Applied Mathematics, 2007).

[53] L.L.Graef, F.E.M.Costa, and J.A.S.Lima, Phys. Lett. B 728, 400 (2014).

[54] I. Brevik, Phys. Rev. D 65, 127302 (2002). 